\title{
Molecular techniques for the detection of the antimicrobial sensitivity: friend or foe?
}

\author{
Vittorio Sambri \\ Unit of Microbiology, The Great Romagna Hub laboratory, Pievesestina and DIMES, University of Bologna, Italy
}

The introduction of molecular techniques into the routine diagnostic workflow of the Microbiology laboratories started some time ago and in the last 10 years this has also included the detection of the antimicrobial sensitivity of bacteria and fungi.

Figure 1 reports the number of published paper per year found in the PubMed web site (https://www.ncbi.nlm.nih.gov/pubmed, accessed on February $5^{\text {th }}, 2018$ ) with the research key antimicrobial susceptibility testing molecular method: it is clearly evident that the research in this field produced an increasing number of papers year after year, with more than 100 manuscripts annually from 2013 to 2017.

This impressive expansion of papers clearly has as a consequence the increase of the routine use of molecular based techniques in the daily workflow for several Microbiology laboratories.

This brief editorial is dedicated to a partial review of what is today available in the field of molecular antimicrobial susceptibility testing (mAST) and to comment on what are the most prominent pros and cons in the use of these methods.

According to the large variety of molecular methods that can be used for the identification of specific microbial genes, these techniques are nowadays widely used to evaluate the presence of target sequences capable to determine antibiotic resistant phenotypes in clinically relevant germs.

The list of the available (most commercially) techniques to perform mAST is very long, but almost all fall within the following categories: PCR based (either with single or multiple target sequences, mostly with real time detection), MALDI-ToF,

Correspondence: Vittorio Sambri, Unit of Microbiology, Centro Servizi AUSL della Romagna, Piazza della Liberazione 60, 47522 Pievesestina (FC), Italy.

Tel.: +39.0547.394906.

E-mail: vittorio.sambri@auslromagna.it

Key words: antimicrobial sensitivity, molecular techniques.

Conflict of interest: the author declares no potential conflict of interest.

Received for publication: 15 February 2018.

Accepted for publication: 15 February 2018.

CC Copyright V. Sambri, 2017

Licensee PAGEPress, Italy

Microbiologia Medica 2017; 32:7346

doi:10.4081/mm.2017.7346

This article is distributed under the terms of the Creative Commons Attribution Noncommercial License (by-nc 4.0) which permits any noncommercial use, distribution, and reproduction in any medium, provided the original author(s) and source are credited. microarrays and FISH, microfluidics and, finally, whole genome sequencing.

In brief, the methods based on the amplification of selected resistance gene by the many available technical variants of PCR are by far the most commonly used in routine. The PCRs techniques have been in place since many years for the identification of single antimicrobial resistance targets, such as the family of genes determining the MRSA/MRSE phenotypes of the vancomycin resistance related genes in Enterococcus spp. Since the last 5 years these techniques have also been made obtainable in the format of multiple PCR in the box, thus very simply allowing the simultaneous detection of a panel of resistance genes in only one testing run. It is indeed of note that the combination of the genes in these panel play an extremely relevant role in term of clinical utility of the results. As an example, the detection of the major five carbapenemase related genes clearly indicate the possible presence of one of the most spread carbapenemase producing Enterobacteria (CPE), since the panel covers the most epidemiologically relevant CPE related sequences worldwide. This is not the case in the mAST for the detection of genes related to the ESbL phenotype, since the galaxy of related genes (and SNPs variants) is well above any possibility of detection even by using a multiplex targeted technique.

Mass spectrometry (MALDI-ToF), aside being today the reference method for bacterial identification, has been proposed to detect spectral modification related to the resistance to selected molecules, such as carbapenemes and vancomycin. This technique is also used to identify the hydrolysis of drugs after incubation in the presence of bacteria suspected to bear a specific resistance phenotype, with variable results.

Microarrays and related hybridization based techniques such as the FISH could be used to identify specific genes based on the binding with complimentary oligonucleotides. One prominent advantage of microarrays in respect to FISH is owed to the fact that this method can assembly onto a microscopic solid support a large number of different nucleotides sequences, thus allowing the multiple detection of thousands of different genes (and SNPs variants) in a single testing run. On the other hand, FISH as a lower multiplexing capability, but requires less sophisticated instrument to be performed.

Bio-engineering and nanotechnologies have recently evolved allowing the size reduction, or better the miniaturization, of several different molecular assay including some method for mAST. These newly developed assays are in general identified as lab on chip and they require an extremely low volume of reagent (in the magnitude of picolitres). Everything required to achieve the final results is incorporated into these miniaturized devices, including bacterial culture system. As far as the fast response is concerned, these methods are really promising since they can provide results a single shift (i.e. 3 to 7 hours). 


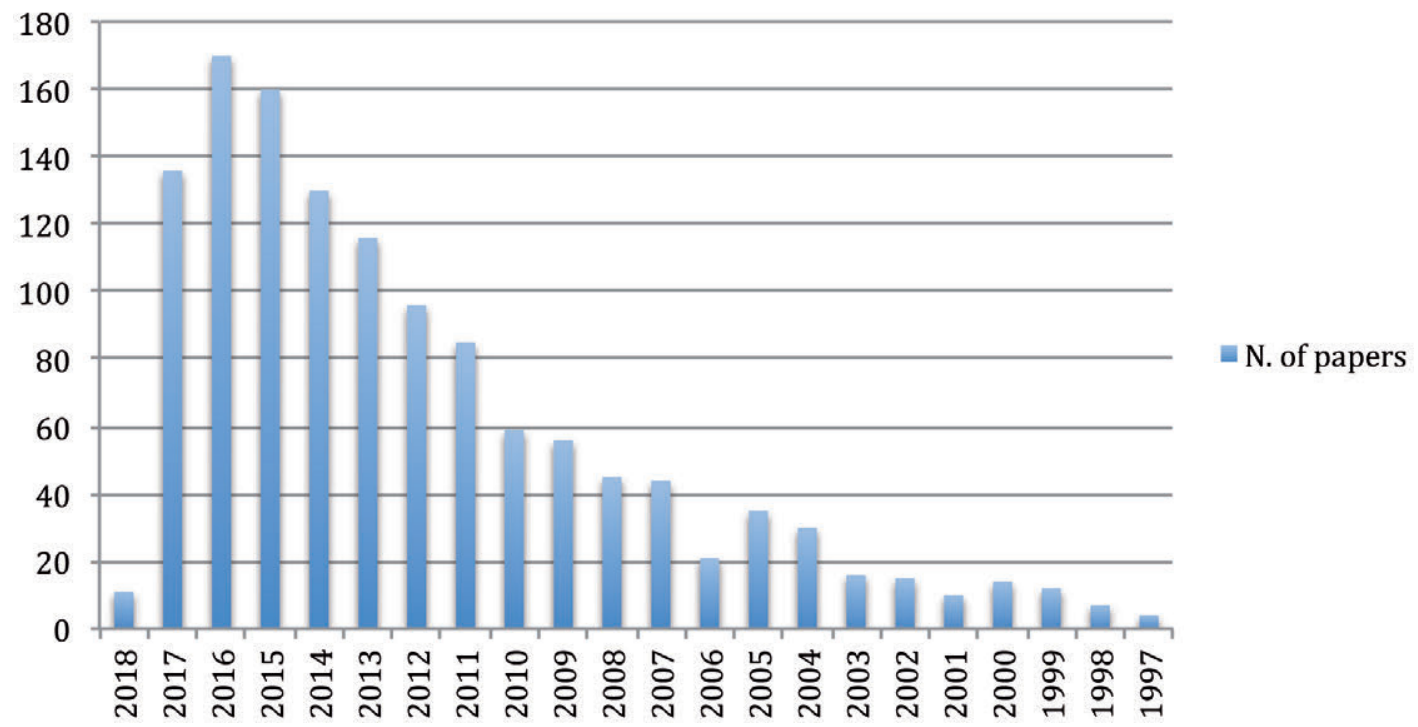

Figure 1. Number of published paper per year found in the PubMed.

The very recent accessibility of next generation sequencing instruments to a larger number of laboratories has brought also the Clinical Microbiologists face to face with the possibility to receive cheap whole bacterial genome sequencing (WGS) data, that of course included also the whole pattern of genes related with antimicrobial resistance. Nowadays there are many pilot studies that show how the WGS data could be useful to identify the pattern of resistance genes, but unfortunately most of these papers are just on small number of clinical isolates and as a consequence the true clinical value of this approach is still to be determined. As very clearly pointed out in the recent paper by E. Carretto (The clinical microbiology in the era of the many-omics, see this issue of Microbiologia Medica) only the joint efforts of well trained Clinical Microbiologists together with skillful Bioinformatics would allow to make the WGS approach a routine clinically relevant tool for the appropriate treatment of severe infections by multidrug resistant germs.

All of the above enlisted diagnostic methods are potentially appropriate on primary blood samples in the case of patients suffering from suspected sepsis, or following the isolation of a germ after a standard culture based protocol.

It is of note that the application of these techniques to the routine microbiology diagnostic workflow has indeed large advantages provided that both the Clinicians and the Microbiologists can interpret the findings with the required level of criticism.

In detail, the most evident pros of this approach to the determination of AST could be summarized as follows: i) germs are not required to be alive or to replicate in vitro (specific gene sequences are detected); ii) very low amount of target sequences are usually identified (each technique has its own limit of detection - LOD largely dependent on the sensitivity of the reaction and on the number of target sequences contained into the reaction mixture itself); iii) the sensitivity of these methods is in general not influenced by any ongoing antibacterial treatment; iv) the turn around time (TAT) is very fast (frequently within one shift time).

The other side of this coin clearly shows some relevant cons as enlisted here: i) these techniques can just detect the presence of predetermined genes, depending on each single panel (see above the issue about the feasibility of a test that detect the ESbL phenotypes); ii) the composition of each panel or the use of tests that identify single target sequences is of course influencing the clinical meaning of the results; iii) the sensitivity of each test is largely depending on the number of targets and on the design of each single PCR reaction; iv) in the case of an unexpected sequence mutation (either at the SNP or at a higher level) the genotype could not be detected even in the presence of a not modified phenotype (i.e. false negative result).

In conclusion, the use of these molecular based techniques for the AST is of certain relevance and will become more and more a common feature of the diagnostic workflow in the clinical Microbiology laboratories. This will generate an undeniable clinical advantage, provided that a precise selection of the patients and of the techniques is achieved.

Who else than a well trained and scientifically updated Clinical Microbiologist could do this? 